\title{
Hafele and Keating on a chip: Sagnac interferometry with a single clock
}

Johnson, Jamie, Foxon, Bethany, Atkocius, Vilius, Gentile, Fabio, Jammi, Sindhu, et al.

Jamie Johnson, Bethany Foxon, Vilius Atkocius, Fabio Gentile, Sindhu Jammi, Konstantinos Poulios, Thomas Fernholz, "Hafele and Keating on a chip: Sagnac interferometry with a single clock," Proc. SPIE 11296, Optical, Opto-Atomic, and Entanglement-Enhanced Precision Metrology II, 1129631 (25 February 2020); doi: 10.1117/12.2552686

SPIE. Event: SPIE OPTO, 2020, San Francisco, California, United States 


\title{
Hafele and Keating on a chip: Sagnac interferometry with a single clock
}

\author{
Jamie Johnson $^{\mathrm{a}}$, Bethany Foxon ${ }^{\mathrm{a}}$, Vilius Atkocius ${ }^{\mathrm{a}}$, Fabio Gentile ${ }^{\mathrm{a}}$, Sindhu Jammi ${ }^{\mathrm{a}}$, \\ Konstantinos Poulios ${ }^{\mathrm{a}}$, and Thomas Fernholz ${ }^{\mathrm{a}}$ \\ aSchool of Physics \& Astronomy, The University of Nottingham, University Park, Nottingham \\ NG7 2RD, UK
}

\begin{abstract}
We describe our progress in the development of an atom based rotation sensor, which employs state-dependent trapping potentials to transport ultracold atoms along a closed path and perform Sagnac interferometry. Whilst guided atom interferometers are sought after to build miniaturized devices that overcome size restrictions from free-falling atoms, fully trapped interferometers also remove free-propagation along an atomic waveguide. This provides additional control of motion, e.g. removing wave-packet dispersion and enabling operation that remains independent of external acceleration. Our experimental scheme relies on radio-frequency and microwave-fields, which are partly generated via atom-chip technology, providing a step towards implementing a small, robust, and eventually portable atomic-gyroscope.
\end{abstract}

Keywords: Atomic sensors, interferometry, inertial sensing, Sagnac interferometer, ring-shaped atom traps, radio-frequency dressed potentials.

\section{INTRODUCTION}

Compared to photons, massive particles possess an advantage for interferometric applications, in that the mass of a typical atom is on the order of $h \nu_{\text {photon }} / m_{\text {atom }} c^{2} \approx 10^{10-11}$ larger than the equivalent mass of a visible photon. ${ }^{1}$ In principle, this ratio can result in a comparable sensitivity gain. However, the challenges in manipulating and coherently guiding atoms with high flux through long interferometer arms are far greater than for photons, which significantly reduces this enhancement. The increase in sensitivity to external influences is simultaneously an advantage and a challenge, and isolating the signal to be measured from all other noise sources is of considerable difficulty. Nevertheless, already demonstrated and further proposed sensing applications of atom interferometers range from fundamental physics to gravitational ${ }^{2}$ and inertial sensors, including rotation sensors that rely on the Sagnac effect. ${ }^{3}$ The most advanced matterwave Sagnac interferometers use free falling atoms and have achieved short-term sensitivities down to $6.0 \times 10^{-10} \mathrm{rad} \mathrm{s}^{-1} / \sqrt{\mathrm{Hz}} \cdot{ }^{4}$ Sustained development has resulted in promising improvements for their use as practical rotation sensors. ${ }^{5}$ The next step in the development of these interferometers is a closed loop waveguide within which atoms can freely propagate ${ }^{6}$ or be locally trapped and transported around the loop. ${ }^{7}$ This achieves independence from free-fall trajectories and confines atoms to a smaller volume that is defined independently of external acceleration. Multiple trips around a closed path may enhance the overall sensitivity to rotation by enclosure of a larger interferometer area without an increase in size. Recent steps in this direction demonstrated coherent transport of a Bose-Einstein condensate over $15 \mathrm{~cm}$ around the circumference of a millimeter sized loop. ${ }^{8}$

\section{AN ATOMIC ROTATION SENSOR}

\subsection{The Sagnac effect}

The classical Sagnac effect as observed for electromagnetic waves, reviewed e.g. by Post, ${ }^{9}$ is purely attributed to a kinematic effect in special relativity and derives from the relativistic law of velocity composition. The Sagnac effect for matterwaves described by the Schrödinger equation does not require a relativistic treatment

E-mail: thomas.fernholz@nottingham.ac.uk

www.coldatomsgroupnottingham.com

Optical, Opto-Atomic, and Entanglement-Enhanced Precision Metrology II, edited by Selim M. Shahriar,

Jacob Scheuer, Proc. of SPIE Vol. 11296, 1129631 - (c) 2020 SPIE

CCC code: $0277-786 \mathrm{X} / 20 / \$ 21 \cdot$ doi: $10.1117 / 12.2552686$

Proc. of SPIE Vol. $112961129631-1$ 
and derives from the fact that the wavefunction is not Galilean invariant. Consider a matterwave moving with a non-relativistic velocity $v^{\prime}$ in an inertial frame $\Sigma^{\prime}$ along the $x^{\prime}$-direction, which is also moving at a velocity $u$ relative to a second inertial frame $\Sigma$. At a time $t$ (common to both frames) the phase of the wave is then given in the Schrödinger theory by $\Phi^{\prime}\left(x^{\prime}, t\right)=\left(p^{\prime} x^{\prime}-E^{\prime} t\right) / \hbar$, where $E^{\prime}$ is the kinetic energy and $p^{\prime}$ the momentum as observed from $\Sigma^{\prime}$. If we describe the same wave from the frame $\Sigma$ using Galilean transformations in the velocity and position, we find there is a correction factor to the phase $\Phi^{\prime}\left(x^{\prime}, t\right)=\Phi(x, t)-m_{0}\left(u x+u^{2} t / 2\right) / \hbar$; this correction factor is not observed in the phase of non-relativistic classical waves. Considering all the Fourier components of a wavefunction $\Psi^{\prime}\left(x^{\prime}, t\right)$ transform in this way, any solution to the Schrödinger equation can then be written as $\Psi^{\prime}\left(x^{\prime}, t\right)=\Psi(x, t) e^{-i m_{0}\left(u x+u^{2} t / 2\right) / \hbar}$. The above discussion is also valid for the case of a one-dimensional path around a loop, as for each instance a momentary inertial frame can be defined for the matterwave whilst it propagates around the loop. This is because all non-inertial forces act perpendicular to the matterwave motion. We can then consider two counter-propagating matterwaves $\Psi_{ \pm}^{\prime}$ moving around a loop fixed in the now non-inertial frame $\Sigma^{\prime}$ in time $2 T$ with equal and opposite angular velocities $\pm \omega_{p}^{\prime}= \pm v^{\prime} / r_{0}$. By substituting in $x_{ \pm}^{\prime}= \pm 2 \pi r_{0}$, where $r_{0}$ is the radius of the loop, the experimenter in the rotating lab frame infers a zero phase difference from the two counter-propagating matterwaves upon completing the full loop. However, from the inertial frame $\Sigma$ it is known that $u=\Omega r_{0}$, where $\Omega$ is the angular velocity of $\Sigma^{\prime}$ and the correction factor $e^{-i m_{0}\left( \pm 2 \pi \Omega r_{0}^{2}+\Omega^{2} t / 2 r_{0}^{2}\right) / \hbar}$ must be included for each matterwave. This then leads to an accumulated phase difference for a single trip around a loop of

$$
\Phi=\frac{4 m_{0} A \Omega}{\hbar} .
$$

Apart from a relativistic correction factor $1 / \sqrt{1-\Omega^{2} r_{0}^{2} / c^{2}}$, where $c$ is the speed of light, and which accounts for the relativistic mass increase, this is the same phase as predicted by relativity. The fact that non-relativistic quantum mechanics can also predict this effect has engendered much discussion. ${ }^{10,11}$ Interestingly, the Sagnac phase is independent of any velocity profile of the quantum particles. As a consequence, it does not only arise for freely propagating waves, but also for trapped particles that are transported around a closed loop. ${ }^{7}$

\subsection{Trapped interferometer scheme}

Our scheme uses trapped particles in state-dependent potentials to form a three-pulse Ramsey interferometer with internal state-labelling. ${ }^{7}$ These states can, for example, be magnetically trappable sub-states of the two hyperfine levels in the electronic groundstate manifold of alkali atoms. The states are chosen such that they experience nearly identical trapping potentials at the interaction points in the sequence, where transitions between the states
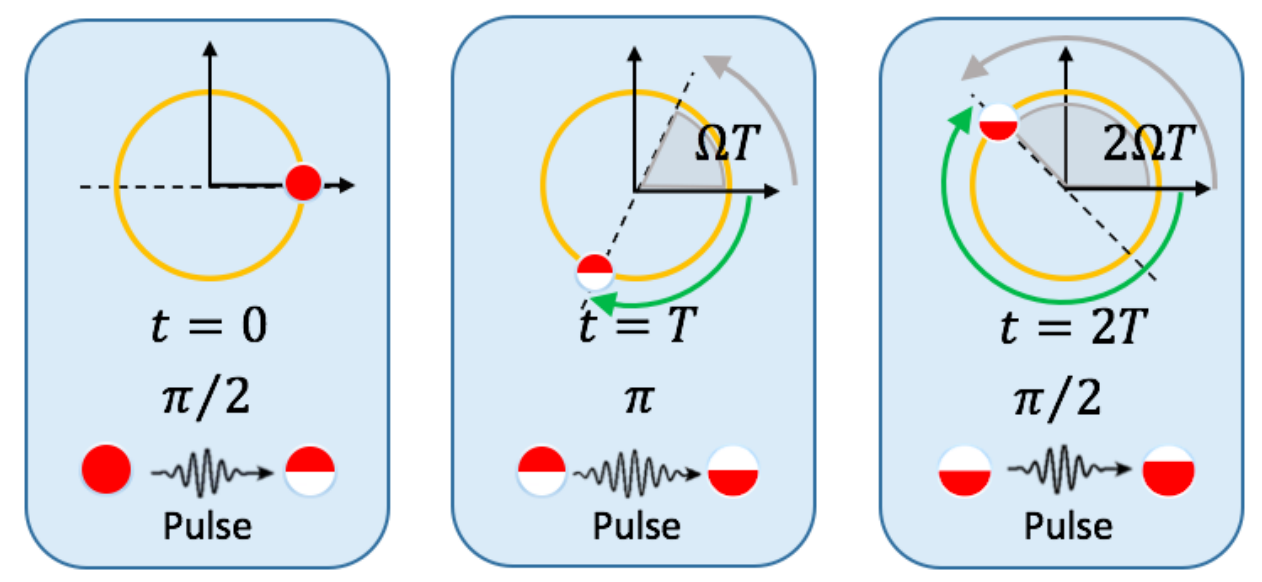

Figure 1. Ramsey sequence for a trapped matterwave interferometer, as seen from an inertial frame $\Sigma$. The first $\pi / 2$-pulse coherently splits the atomic cloud, the $\pi$-pulse reverses the accumulation of dynamical phase between the two spin states, and the final $\pi / 2$-pulse converts the interferometer phase into a population imbalance. The cloud's trajectory is shown for one part of the generated superposition, which does not appear to complete a full cycle due to rotation of the laboratory frame. 
can then be driven by a microwave (MW) field. In contrast to Raman laser-based beamsplitting operations, where opposite photon momenta are imparted onto parts of the quantum mechanical superposition, counterpropagating motion of atoms is induced and controlled via the state-dependence of the potentials. As a result, the interferometer is operated akin to an atomic clock where the clock states are transported between Ramsey pulses. Introducing a central $\pi$-pulse leads to a cancellation of the dynamical clock phases accumulated during

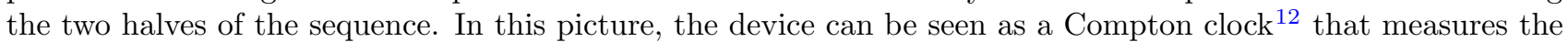
propertime difference for the two paths, a relativistic effect that has indeed been observed with regular atomic clocks by Hafele and Keating. ${ }^{13}$

The interferometric three-pulse sequence and the effect of external rotation is sketched in Fig. 1. Atoms are initialised in the $|\downarrow\rangle$ state (population shown in red). At time $t=0$ a $\pi / 2$-pulse drives the population into a coherent superposition of the two states $|\Psi\rangle=(|\downarrow\rangle+|\uparrow\rangle) / \sqrt{2}$. The magnetically trapped atoms are then state dependently driven in a counter propagating fashion for a half revolution around a ring track in a time $T$, after which a $\pi$-pulse is applied to swap the internal spin states of the atoms. The motion of the state-dependent traps is then symmetrically reversed in the same time $T$, ensuring that atoms spend time $T$ in each spin state whilst traversing the entire loop. After the second half revolution, the interferometer is closed with a final $\pi / 2$-pulse. In the ideal scenario, any dynamical phase is cancelled and only the accumulated Sagnac phase is converted into a population imbalance $\sigma_{z}$ of the two internal states, measured by the expectation value $\left\langle\sigma_{z}\right\rangle=C \cos \left(\Phi-\Phi_{\text {ref }}\right)$. The introduced reference phase $\Phi_{\text {ref }}$ is adjustable via the phase of the final $\pi / 2$-pulse, and can be used to measure with maximal sensitivity at any rotation rate $\Omega$. Corrections to the interferometer phase and a reduced contrast $C$ can be expected to arise from dynamical effects, ${ }^{14}$ decoherence, technical imperfections, and reduced overlap of the recombined wavefunctions.

The key experimental requirements to implement the envisioned scheme are: a pair of trappable states, state-dependent transport of these states, coherent driving of a transition between these states, and sufficient coherence time to maintain a superposition over the cycle time $2 T$. In our experiment we use two magnetically trappable sub-states of the radio frequency (RF) dressed hyperfine ground state manifold of ${ }^{87}$ rubidium with labels $|1,-1\rangle \equiv|\downarrow\rangle$ and $|2,1\rangle \equiv|\uparrow\rangle$. We have demonstrated that state-dependent traps can be formed on a loop for these states with RF-dressed potentials in different configurations. ${ }^{15}$ In the following, we briefly review a configuration that is suitable for an atom-chip based approach, discuss microwave transitions between the relevant states, as well as measures to achieve coherence, and we give some experimental details on our progress towards the realisation of a small scale device.

\section{RADIO-FREQUENCY DRESSED ATOMS}

\subsection{Magnetic trapping potentials}

Standard ${ }^{87}$ rubidium clocks and state-labelled interferometers employ the transition $|1,0\rangle \leftrightarrow|2,0\rangle$, because within the weak field regime the state energies depend only quadratically on a static magnetic field, therefore providing a narrow and stable linewidth for precise and accurate measurement. The magnetically trappable states $|1,-1\rangle$ and $|2,1\rangle$ also provide a narrow and stable two-photon transition at a magic field of $3.23 \mathrm{G}$ where the differential Zeeman-shift vanishes to first order. ${ }^{16}$ Appropriate state dependence potentials can, for example, be introduced by microwave dressing, which has also been proposed for trapped interferometry. ${ }^{17}$ Here we use radio-frequency dressed potentials to introduce additional design freedom and properties, although additional issues also arise which need to be addressed. An important motivation is the generation of ring and hollow-torus shaped traps ${ }^{18}$ and lattices ${ }^{19}$ where state-dependent control and simultaneous operation of oppositely oriented Sagnac interferometers is achieved simply via RF-phase changes. Additional aspects include the possibility of low-noise dispersive detection, ${ }^{20}$ and also a complex spectrum of hyperfine multi-photon transitions that combine RF-photons from the dressing fields with additional microwave driving. ${ }^{21}$

The principle of RF-dressed potentials ${ }^{22}$ is to combine static $\mathbf{B}_{\mathrm{DC}}$ with time-dependent $\mathbf{B}_{\mathrm{RF}}(t)$ magnetic fields that drive atomic spin-flips. Neglecting the internal structure of an atom and within the weak field regime, the Hamiltonian for an atom with total spin F interacting with a static and single-frequency RF-field of arbitrary polarisation is given by

$$
\mathrm{H}=\mu_{\mathrm{B}} g_{\mathrm{F}} \mathbf{F} \cdot\left(\mathbf{B}_{\mathrm{DC}}+\frac{\mathbf{B}_{\mathrm{RF}}(t) e^{i \omega_{\mathrm{RF}}}+\text { c.c. }}{2}\right),
$$


where $g_{\mathrm{F}}$ is the Lande $g$-factor, $\mu_{\mathrm{B}}$ the Bohr-magneton, and $\mathbf{F}$ the angular momentum operator in units of $\hbar$. A static field $\mathbf{B}_{\mathrm{DC}}=B_{\mathrm{DC}} \hat{\mathbf{e}}_{0}$ of magnitude $B_{0}$ along unit vector $\hat{\mathbf{e}}$ lifts the degeneracy of Zeeman sublevels and defines a quantization axis that may be spatially dependent. The time dependent field $\mathbf{B}_{\mathrm{RF}}(t)$ of frequency $\omega_{\mathrm{RF}}$ couples the Zeeman levels, causing transitions between the $m_{\mathrm{F}}$ states. For an atom traversing a region of resonance $\omega_{\mathrm{RF}}=\mu_{\mathrm{B}} m_{\mathrm{F}} g_{\mathrm{F}}\left|\mathbf{B}_{\mathrm{DC}}\right| / \hbar$ with sufficient coupling strength, adiabatic transfer will occur between high and low-field seeking states such that the atom may be trapped in the resonant region. The coupling strength is given by a circularly polarised component of the RF-field that drives spin-precession about the spatially dependent field direction. It is easily evaluated by constructing a local basis, choosing a unit vector $\hat{\mathbf{e}}_{1}$ orthogonal to $\hat{\mathbf{e}}_{0}$, and completing the basis with $\hat{\mathbf{e}}_{2}=\hat{\mathbf{e}}_{0} \times \hat{\mathbf{e}}_{1}$. Projection of the RF-field onto this basis provides spherical components $\mathbf{B}_{\mathrm{RF}}=\beta_{+} \hat{\mathbf{e}}_{+}+\beta_{-} \hat{\mathbf{e}}_{-}+\beta_{0} \hat{\mathbf{e}}_{0}$, with $\hat{\mathbf{e}}_{ \pm}=\left(-\hat{\mathbf{e}}_{1} \pm i \hat{\mathbf{e}}_{2}\right) / \sqrt{2}$. In the rotating wave approximation (RWA), the adiabatic potentials of the dressed states, i.e. the spectrum of quasi-energies, is found to be

$$
E_{\mathrm{F}, \bar{m}}=\mu_{\mathrm{B}} \bar{m} g_{\mathrm{F}} \sqrt{\left(\left|\mathbf{B}_{\mathrm{DC}}\right|-\frac{\hbar \omega_{\mathrm{RF}}}{\mu_{\mathrm{B}}\left|g_{\mathrm{F}}\right|}\right)^{2}+\frac{\left|\beta_{ \pm}\right|^{2}}{2}},
$$

where the sign of the relevant component $\beta_{ \pm}$is equal to that of the $g_{F}$-factor for $\omega_{\mathrm{RF}}>0$. The Rabi frequency of resonant coupling is given by $\hbar \Omega_{\mathrm{RF}}=\mu_{B} g_{\mathrm{F}} \beta_{ \pm} / \sqrt{2}$. The RWA is generally valid for $\Omega_{\mathrm{RF}} \ll \omega_{\mathrm{RF}}$, and adiabaticity across the resonance this requires that $\omega_{0} \ll \Omega_{\mathrm{RF}}$, where $\omega_{0}$ is the angular trap frequency.

The three-dimensional shape of the resulting potentials is determined by two parts. The first expression under the radical in Eq. 3 is solely frequency dependent and usually dominant, such that it defines a resonant surface to which atoms are confined. The second expression is solely polarisation dependent and shapes the potential within the resonant surface. Suitable construction of $\mathbf{B}_{\mathrm{DC}}$ and $\mathbf{B}_{\mathrm{RF}}(t)$ can then result in highly controllable atom trapping potential landscapes.

\subsection{Multipoles and ring shaped lattices}

A natural choice of potential landscape we can exploit for a matterwave Sagnac interferometer is one of axial symmetry, which is able to locally trap atoms and state-dependently transport them around a ring. Following our
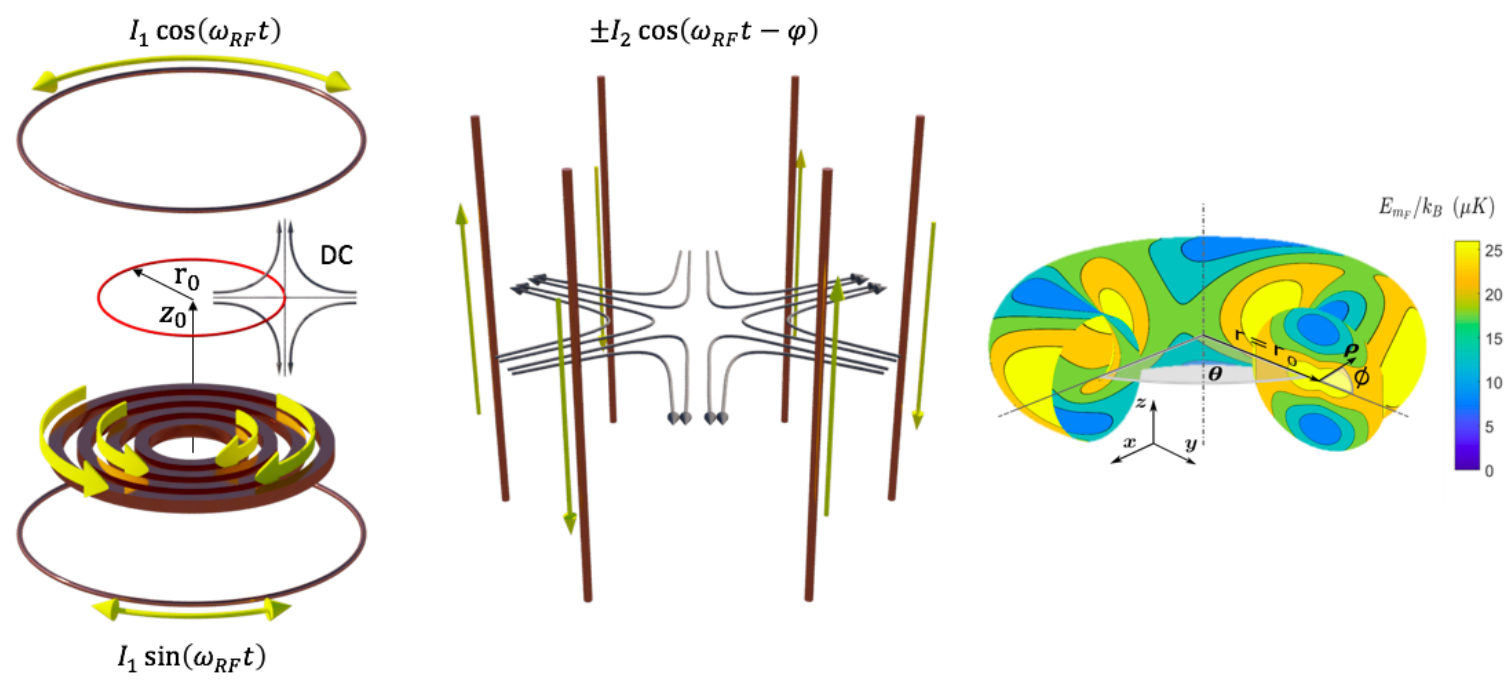

Figure 2. The leftmost sketch shows four concentric coils in the $x-y$ plane with counterpropagating DC currents (flat yellow arrows). These coils create a static magnetic ring quadrupole with a radius $r_{0}$ and at a height $z_{0}$. The central ring of zero-field is depicted as a red circle. Two RF-coils shown above and below may create the field $\mathbf{B}_{\mathrm{RF}}^{(t)}(t)$. They each carry RF current of magnitude $I_{1}$, but are operated out-of-phase, such that traps are created at the top and bottom of the torus potential i.e. at poloidal angles $\phi= \pm \pi / 2$. The middle figure displays a particular multipole field $\mathbf{B}_{\mathrm{RF}}^{(m)}(t)$ : the $l=3$ hexapole, which can be created by 6 pillars of counterpropagating AC currents shown by the vertical yellow arrows. This field interferes with $\mathbf{B}_{\mathrm{RF}}^{(t)}(t)$ to create the potential sketched in the rightmost figure, depicting the resonant surface and a vertical cut through the potential landscape. 
trapping scheme described in, ${ }^{19}$ we start with an axially symmetric static field in the shape of a ring quadrupole. Such a field can be created with four concentric, counter-propagating currents as shown in Fig. 2, which produces a ring of zero field with radius $r_{0}$ at a distance $z_{0}$. In the vicinity of the ring, the magnitude of this static field increases linearly with distance $B_{\mathrm{DC}}=q \rho$, where $q$ is the ring-quadrupole gradient and $\rho$ the distance from the field zero. Using the direction $\mathbf{e}_{0}$ of the static field, approximated for $\rho \ll z_{0}, r_{0}$, a local, Cartesian basis can be constructed according to

$$
\begin{aligned}
& \mathbf{e}_{0}=(-\cos \theta \cos \phi,-\sin \theta \cos \phi, \sin \phi)^{T} \\
& \mathbf{e}_{1}=(-\sin \theta, \cos \theta, 0)^{T}, \\
& \mathbf{e}_{2}=(-\cos \theta \sin \phi,-\sin \theta \sin \phi,-\cos \phi)^{T}
\end{aligned}
$$

where $\mathbf{e}_{1}$ is a unit vector tangent to the ring, and $\theta$ and $\phi$ are toroidal and poloidal angles, respectively. An RF-field of frequency $\omega_{\mathrm{RF}}$ resonantly couples on a toroidal surface of minor radius $\rho_{0}=\hbar \omega_{\mathrm{RF}} / \mu_{\mathrm{B}}\left|g_{\mathrm{F}}\right| q$ and major radius $r_{0}$. A suitable choice of RF-field polarisation can then be used to modulate the corresponding potential on the toroidal surface. We introduce a phase-shifted combination of a vertical $(z)$ and approximately radial $(r)$ RF-field, as this can be generated near the central plane of a horizontal coil pair, see Fig. 2. Such a dressing field provides non-zero coupling strength across the entire toroidal surface (t) and maintains axial symmetry. It can be written in terms of complex circular amplitudes $a_{ \pm}$, which we assume to be constant in the vicinity of the forming trap. The field is then expressed as

$$
\mathbf{B}_{\mathrm{RF}}^{(\mathrm{t})}=\frac{a_{+}}{\sqrt{2}}\left(\begin{array}{c}
\cos \theta \\
\sin \theta \\
i
\end{array}\right)+\frac{a_{-}}{\sqrt{2}}\left(\begin{array}{c}
\cos \theta \\
\sin \theta \\
-i
\end{array}\right) .
$$

For the case of a single circular component, i.e. $a_{+}=0$ or $a_{-}=0$, the coupling strength $\left|\beta_{ \pm}\right|=\left|\hat{\mathbf{e}}_{ \pm} \cdot \mathbf{B}_{\mathrm{RF}}^{(t)}\right|=$ $\left|\left(-a_{+} e^{-i \phi}+a_{-} e^{i \phi}\right)\right| / 2$ becomes independent of both $\theta$ and $\phi$, resulting in a homogeneous potential over the resonant toroidal surface. For our interferometer we are interested in the case where poloidal minima form at both the top and bottom of the torus. This can be achieved in the case of an elliptical field, $0<\left|a_{+}\right| \neq\left|a_{-}\right|>0$, where the homogeneous potential splits into two poloidal minima when the radial field $a_{r}=\left(a_{+}+a_{-}\right) / \sqrt{2}$ is smaller in magnitude than the vertical RF-component $a_{z}=\left(a_{+}-a_{-}\right) / \sqrt{2}$. and the corresponding real fields have a $90^{\circ}$ phase difference. A state dependent potential modulation within these rings can now be achieved with a second RF-field at the same frequency $\omega_{\mathrm{RF}}$ and polarised in the $x-y$ plane. For multiple minima within a ring, we consider a multipole RF-field $\mathbf{B}_{\mathrm{RF}}^{(\mathrm{m})}$ of order $l$, which will conveniently interfere with the axially symmetric field $\mathbf{B}_{\mathrm{RF}}^{(\mathrm{t})}$. This creates toroidal minima where atoms can be localised around the rings. For simplicity, we describe such a field as an inner multipole, which approximates the field inside a ring of vertical currents of alternating orientation. The idealised case for a hexapole with $l=3$ is depicted in Fig. 2.

Decomposed into circular components, the cylindrical multipole field $(\mathrm{m})$ can be expressed in terms of its circular components as ${ }^{19}$

$$
\mathbf{B}_{\mathrm{RF}}^{(\mathrm{m})}=\frac{\mathcal{U}_{+}}{\sqrt{2}}\left(r e^{-i \theta}\right)^{l-1}\left(\begin{array}{l}
i \\
1 \\
0
\end{array}\right)+\frac{\mathcal{U}_{-}}{\sqrt{2}}\left(r e^{i \theta}\right)^{l-1}\left(\begin{array}{c}
i \\
-1 \\
0
\end{array}\right),
$$

where $\mathcal{U}_{ \pm}$are the complex moments. Again neglecting the spatial variation of this field in the vicinity of the forming trap, we can approximate the field amplitudes locally as $u_{ \pm}=\mathcal{U}_{ \pm} r_{0}^{(l-1)}$. Evaluating the joint circular component $\beta_{ \pm}=\hat{\mathbf{e}}_{ \pm} \cdot\left(\mathbf{B}_{\mathrm{RF}}^{(\mathrm{t})}+\mathbf{B}_{\mathrm{RF}}^{(\mathrm{m})}\right)$, a ring lattice structure emerges from the interference. Assuming real amplitudes $a_{ \pm}$for the toroidal RF-field, and considering only the coupling at the top and the bottom of the torus, we find for atoms with positive $g_{F}$-factor

$$
\left|\beta_{+}\right|_{\phi= \pm \pi / 2}^{2}=\frac{1}{2} a_{r}^{2}+\left|u_{ \pm}\right|^{2} \pm \sqrt{2} a_{r}\left|u_{ \pm}\right| \sin \left(l \theta \mp \varphi_{ \pm}\right),
$$

where we introduced the phases of the circularly polarised multipole components according to $u_{ \pm}=\left|u_{ \pm}\right| e^{i \varphi_{ \pm}}$. 
Equation 9 clearly shows modulations of the potential around the top and bottom of the torus, which are independently controlled by the multipole components. The number of minima is equal to the multipole order $l$ and is a consequence of the winding number difference of the two RF-fields around the ring path. The locations of the minima in the top and bottom rings are independently controlled by the two phases $\varphi_{ \pm}$. Figure 2 shows the resulting potential landscape on the resonant surface, and a cut through the torus body for an $l=3$ multipole field with $a_{r}=0.9 \mathrm{G}, a_{z}=1.5 \mathrm{G},\left|u_{ \pm}\right|=0.3 \mathrm{G}$. It should be noted that for negative $g_{F}$ the role of the field amplitudes $\left|u_{ \pm}\right|$will be swapped, which introduces the state-dependent control of the modulation that is required to operate a trapped Sagnac interferometer. Such interferometers will then be formed in pairs, one in each ring, but with opposite rotational senses.

\subsection{Radio-frequency dressed hyperfine transitions}

In order to operate a clock-like, trapped Sagnac interferometer with RF-dressed alkali atoms, the structure of the dressed hyperfine spectrum must be understood. The presence of the dressing field enables multi-photon transitions, which leads to some complexity. This allows for microwave transitions between hyperfine levels that are forbidden by standard selection rules in the undressed case, leading to pseudo-one-photon transitions between any pair of dressed levels. Another consequence is the emergence of groups of transitions to quasienergy levels that are separated by multiples of the RF-dressing frequency, $\omega_{\mathrm{RF}} \cdot{ }^{21}$ Each group occurs near one of the seven possible bare transition frequencies, and these reflect the standard selection rules for microwave polarisation. Even numbered groups $n=0, \pm 2$ may be addressed with $\pi$-polarised MW, and odd numbered groups $n= \pm 1, \pm 3$ with $\sigma$-polarisation. However, all groups permit the MW probing of each of the transitions $|1, \bar{m}\rangle \leftrightarrow\left|2, \bar{m}^{\prime}\right\rangle$. Within each group, for each level $|1, \bar{m}\rangle$, there are five possible transitions separated at intervals of the RF Rabi frequency, some of which become forbidden for certain experimental parameters.

In order to take spectroscopic data we prepare a pure $|1, \bar{m}\rangle$ state, and then apply a MW probe to transfer population into $\mathrm{F}=2$. Fig. 3 shows an experimental hyperfine spectrum for ${ }^{87}$ rubidium, prepared in the resonantly dressed $|1,-1\rangle$ state at a radio-frequency of $180 \mathrm{kHz}$. A dispersive detection technique was used, ${ }^{20}$ and the peak heights are not indicative of transition strength, but signify birefringent properties of the targeted state in the $\mathrm{F}=2$ manifold, together with resulting population. A zoom into one of the groups $(n=1)$ reveals

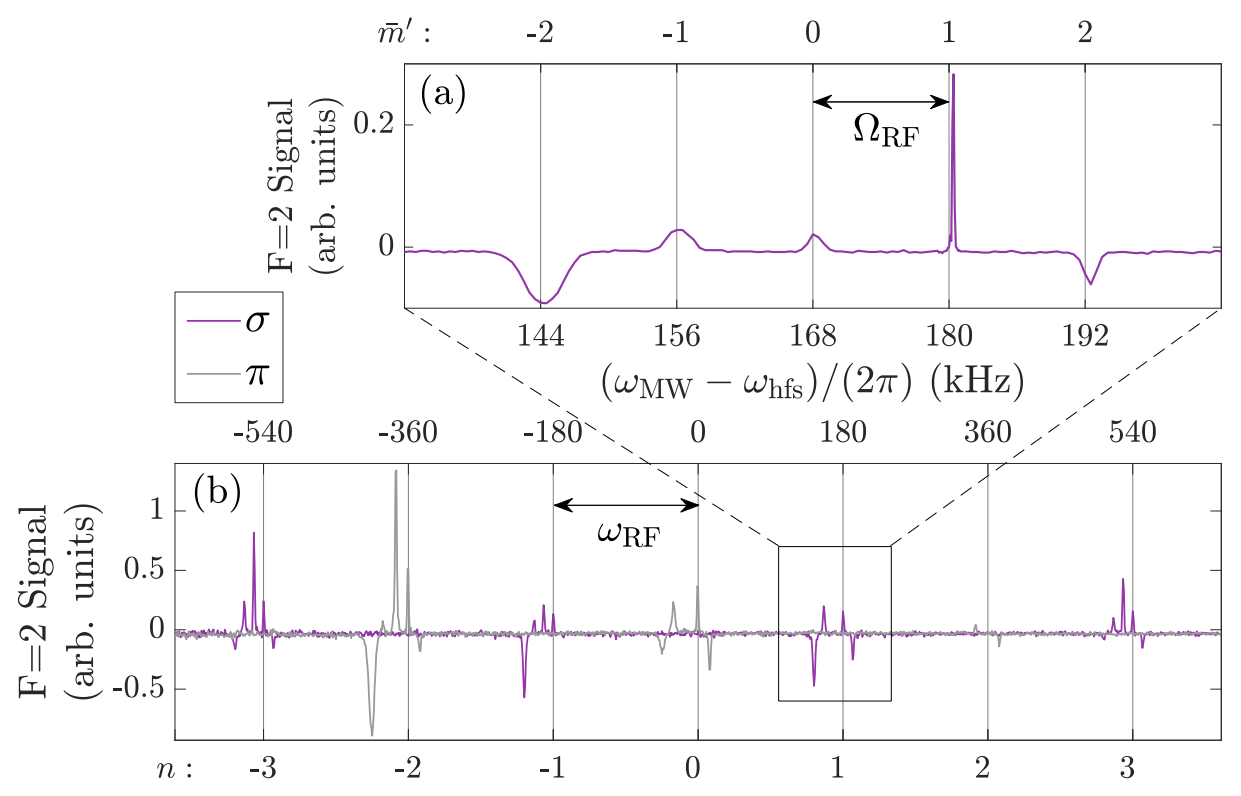

Figure 3. Spectroscopic data of the MW transitions $|1,-1\rangle \rightarrow\left|2, \bar{m}^{\prime}\right\rangle$ in the $n=1$ group (a) and all groups (b). The narrow line-width pseudo-clock transition $|1,-1\rangle \rightarrow|2,1\rangle$ used in the interferometer scheme can be seen in (a). The transitions occur at the frequencies given by $\omega_{\mathrm{hfs}}+n \omega_{\mathrm{RF}}+\bar{m} \Omega_{\mathrm{RF}}^{-}+\bar{m}^{\prime} \Omega_{\mathrm{RF}}^{+}$, where $\bar{m}=-1$, $\omega_{\mathrm{RF}}=180 \mathrm{kHz}$ and $\Omega_{\mathrm{RF}}^{ \pm}=12.0 \pm 0.2$ $\mathrm{kHz}$ (with corresponding $\beta^{ \pm}=24.1 \pm 0.4 \mathrm{mG}$ ). 
transitions to all five target states. The sharp line at a detuning of $180 \mathrm{kHz}$ from the hyperfine frequency $\omega_{\mathrm{hfs}}=$ $2 \pi \times 6.834 \mathrm{GHz}$ corresponds to the transition of interest, namely between the trapped states $|1,-1\rangle \rightarrow|2,1\rangle$, which have nearly vanishing differential energy difference as a function of external magnetic field strength.

\section{COHERENT DRIVING AND COHERENCE CONTROL}

\subsection{A dressed pseudo-clock transition}

In the following, we give further details on the transition between the two magnetically trappable, dressed states $|1,-1\rangle$ and $|2,1\rangle$. This transition is comparatively sharp, but still depends on external parameters. The transition frequency can be made precise, i.e. resulting in a narrow linewidth in the presence of field fluctuations, as well as in a trapped scenario where external fields spatially vary. The frequency is not necessarily accurate however, and we therefore refer to it as a pseudo-clock transition. The three-pulse interferometric sequence is nonetheless robust against inaccuracy, as seen below.

As a proof of principle we applied the interferometric sequence using these dressed pseudo-clock states to untrapped atoms in freefall. ${ }^{23}$ Atoms are initially prepared in the bare state $\left|1, m_{\mathrm{F}}=-1\right\rangle$ and adiabatically dressed by tuning into RF-resonance. We then perform the full three-pulse Ramsey sequence using MW with near-resonant frequency $\omega_{\mathrm{MW}}$ on a transition to the dressed state $\left|2, \bar{m}_{\mathrm{F}}=1\right\rangle$. The first two MW pulses are of the same phase, whilst the phase of the final pulse differs by $\Phi_{\text {ref }}$. The entire spin-echo sequence can be modelled in terms of unitary evolution operators as $|\Psi(2 T)\rangle=U_{\pi / 2}\left(\Phi_{\text {ref }}\right) U_{\uparrow \downarrow}(T) U_{\pi}(0) U_{\uparrow \downarrow}(T) U_{\pi / 2}(0)|\downarrow\rangle$, where $U_{\uparrow \downarrow}$ describes the free evolution of the internal spin states in the external fields, and $U_{\theta}(\phi)$ describes MW driving pulses with area $\theta$ and phase $\phi$. Scanning both $\Phi_{\text {ref }}$ and the detuning from resonance $\Delta$ results in the data in Fig. 4a. The colour map indicates the population transfer, which is in good agreement with the theoretical model shown in Fig. 4b. Panel (c) shows model and data with a sinusoidal fit for the interferometric fringe obtained at $\Delta=0$, corresponding to the dashed lines in (a) and (b). A small phase offset of $\approx 0.38$ rad can be seen, which we attribute to field inhomogeneities that the atoms experience during their free fall. Local MW phase differences, as well as a small time-dependent shift in atomic transition frequency, will both result in such an effect. The experimental data also shows that the nominal zero-detuning was in fact finite but within the robustness region, such that the interferometric fringe remains largely unaffected.

In the following, we discuss two issues that will affect the linewidth in the trapped scenario and envisioned remedies. The first effect arises from the influence of the magnetic moment of the atomic nucleus, and the second effect from the handedness of RF-field geometry in the vicinity of the trap minima. Both effects can be expressed

(a)

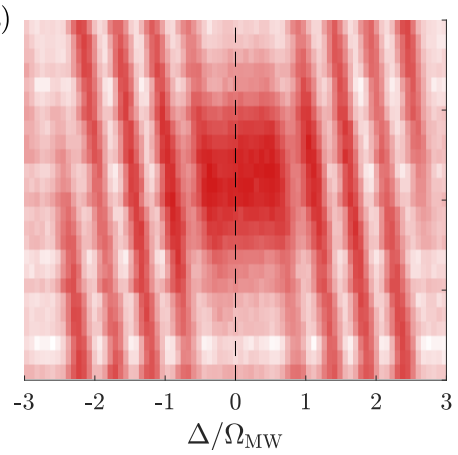

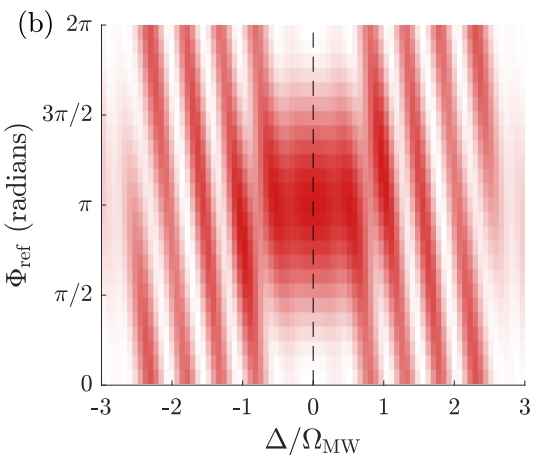
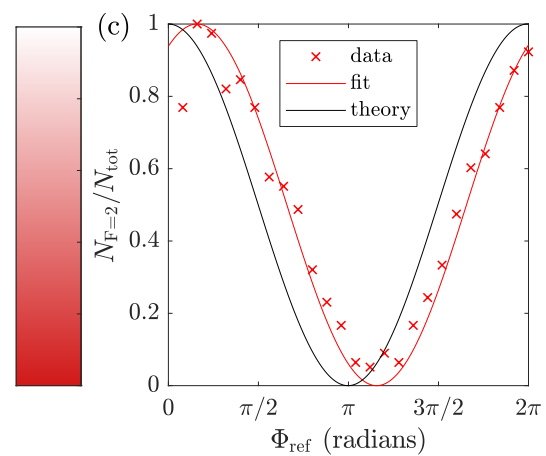

Figure 4. Interferometry data for RF-dressed, free falling atoms is shown in (a) as a colour map of the population fraction in the $\mathrm{F}=1$ level $\left(N_{\mathrm{F}=1} / N_{\text {tot }}\right)$ after the full three-pulse Ramsey sequence. Atoms begin in $|1,-1\rangle$, and the axes show the MW detuning from resonance for the transition to $|2,1\rangle$ (normalised by the Rabi frequency of the driving $\Omega_{\mathrm{MW}}$ ), and the phase difference $\Phi_{\text {ref }}$ of the final MW-pulse. The normalised sequence timing is $T \Omega_{\mathrm{MW}}=7.5$. In (b) we show the theoretical prediction of the same data, as given by a full treatment of the evolution operators and a measured Rabi frequency of $\Omega_{\mathrm{MW}}=540 \pm 4 \mathrm{~Hz}$. This shows good agreement with the data. In (c) both the theoretical (black) and measured (red) population fraction at zero-detuning are shown, with a fit to the data which illustrates the slight offset in phase. This plot corresponds to the data and theory in (a) and (b) at the dashed lines. 
as a small mismatch between trapping potentials for the two states, which leads to a locally dependent shift of transition frequency. As discussed by Gentile, ${ }^{23}$ trap misalignment affects wavefunction overlap between the two internal states, and results in heating of the atomic sample during beam splitter operations and reduced contrast in the final signal.

\subsection{Nuclear magnetic moment and microwave dressing of RF-dressed states}

The two trapped states are chosen from different ground state hyperfine manifolds due to the opposite signs of their Landé-factors $g_{\mathrm{F}}$. This alters the sense of precession of the total spin in a magnetic field, which enables state-dependent control via circularly polarised RF-fields of opposite rotational senses. When atoms interact with the interferometric driving pulses, the trapping potentials for the two states should ideally be identical. In the described scenario, this is possible for Landé factors of equal magnitude. However, the nuclear magnetic moment, which changes orientation with respect to the dominating electronic moment between hyperfine levels, introduces a small difference in magnitude $\left(g_{1} \approx-0.5018\right.$ and $\left.g_{2} \approx 0.4998\right)$ and thus a shift in RF resonance. The result is a small but significant displacement between RF-dressed traps along the gradient of static field modulus, and equivalently a static field dependent linear shift of the pseudo-clock transition frequency.

In order to cancel this dependence we make use of the AC Zeeman effect, which shifts state energies in the presence of a detuned driving field. For different RF-dressing arrangements the use of MW-dressing for such purposes has been studied. ${ }^{24}$ The principle adapted to our scenario is sketched in Fig. 5 in the dressed state picture. Apart from the targeted pseudo-clock transition, several other transitions $|1, \bar{m}\rangle \rightarrow\left|2, \bar{m}^{\prime}\right\rangle$ are possible, with quasi-energies

$$
E_{1, \bar{m} \rightarrow 2, \bar{m}^{\prime}}=\hbar \omega_{\mathrm{hfs}}+n \hbar \omega_{\mathrm{RF}}+E_{2, \bar{m}^{\prime}}-E_{1, \bar{m}}
$$

where $n$ ranges from -3 to 3 for ${ }^{87} \mathrm{Rb}$. Two groups of these quasi-energy levels are indicated with dashed lines in Fig. 5.

By introducing off-resonant driving to shift one of the target states and leave the other largely unaffected, the transition frequency can be altered, and we wish to introduce such a shift that exactly cancels the static field shift. To lowest order, and considering only a two level system, the AC-Zeeman shift will be $\Delta E(B) \approx$ $\Omega_{\mathrm{MW}}^{2}(B) / \Delta_{\mathrm{MW}}(B),{ }^{25}$ where $\Omega_{\mathrm{MW}}$ and $\Delta_{\mathrm{MW}}$ are the Rabi frequency and detuning for the off-resonantly driven transition. It is important to note, however, that near a dressed trap minimum all dressed state energies and thus any detuning $\Omega_{\mathrm{MW}}$ necessarily depend quadratically on the static field strength, see Eq. 3. A linear shift

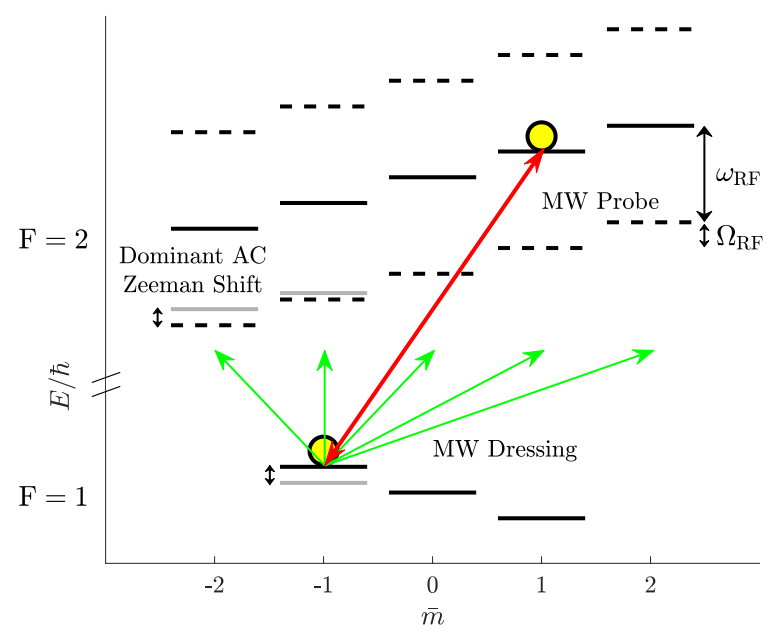

Figure 5. Energy level diagram sketching the effect of the AC Zeeman shift on the RF-dressed hyperfine levels. The probe transition of interest $|1,-1\rangle \leftrightarrow|2,1\rangle$ is labelled in red, with the energy levels of the probed group shown as solid lines. The MW-dressing is shown in green and addresses another group of quasi-energy levels, indicated by dashed lines. The dominant AC Zeeman shift stems from the transition to the $|2,-2\rangle$ level as labelled, causing shifts (grey) on the original (black) energy levels. Smaller, detuning-dependent line shifts will occur on all other quasi-energy levels, but will be negligible in most groups. 

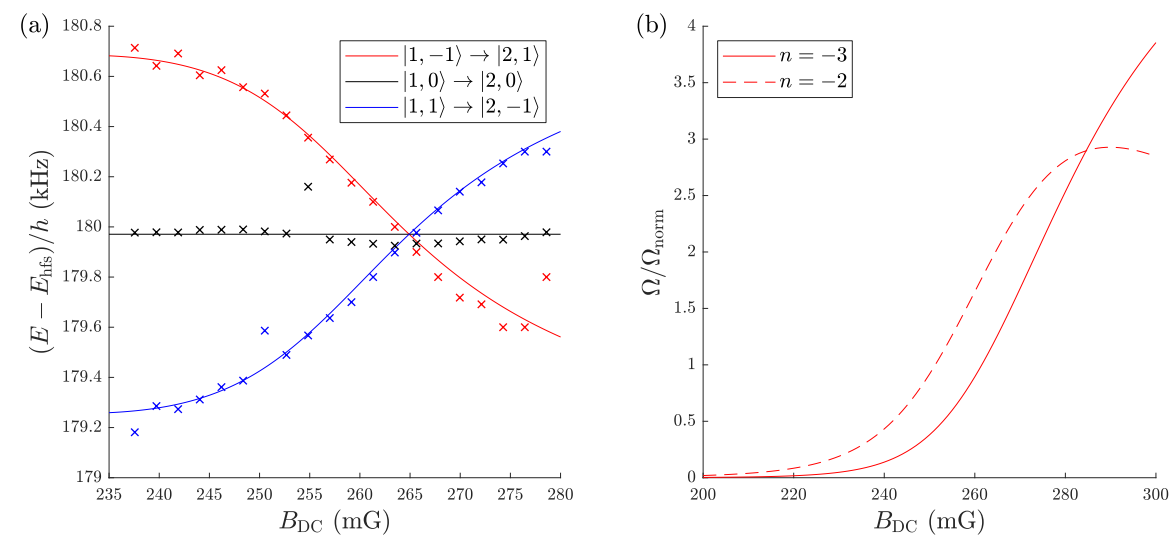

Figure 6. The static magnetic field dependence of the pseudo-clock transitions in group $n=1$ is shown in (a) for $B_{\text {res }}=257$ $\mathrm{mG}$ and $\omega_{\mathrm{RF}}=180 \mathrm{kHz}$, with model fits according to Eq. 3, obtaining the value $\beta_{ \pm}=26 \pm 3 \mathrm{mG}$. This is consistent with our value $\beta=24.1 \pm 0.4$ from the data in Fig. 3. The (pseudo-)clock transitions from $m=0, \pm 1$ are shown in red, black and blue respectively. Using the same field magnitudes, the theoretical Rabi frequencies $\Omega_{\mathrm{MW}}$ of the transition $|1,-1\rangle \rightarrow|2,-2\rangle$ in groups $n=-3$ (solid line) and $n=-2$ (dashed line) are shown in (b). The normalisation factor $\Omega_{\text {norm }}$ accounts for the proportionality to MW dressing field amplitude.

can therefore only be cancelled via a static field dependent Rabi frequency. Due to the opposite sign of $g_{\mathrm{F}}$-factors most dressed hyperfine transitions show such a dependence. ${ }^{21}$

In order to determine the necessary microwave dressing to reduce the static field dependence we must first know the shape of the dependence we wish to cancel. We investigated this with free falling atoms in homogeneous fields. Figure 6a shows the measured frequencies for all three transitions $|1, \bar{m}\rangle \leftrightarrow|2,-\bar{m}\rangle$ in group $n=1$ as a function of static magnetic field. Each data point was extracted from frequency scans and model fits to the transferred population after $\mathrm{MW}$ probing. For the used parameters $\left(B_{\mathrm{DC}}=257 \mathrm{mG}, B_{\mathrm{RF}} \approx 26 \mathrm{mG}\right)$ a static field change of $\approx 0.8 B_{\mathrm{DC}}$ causes a frequency shift of $\approx 0.5 \mathrm{kHz}$.

We can now consider the form of dressing required to cancel this dependence. The dressing frequency should be sufficiently far from any resonance that would lead to undesired population transfers. Accounting for this, we
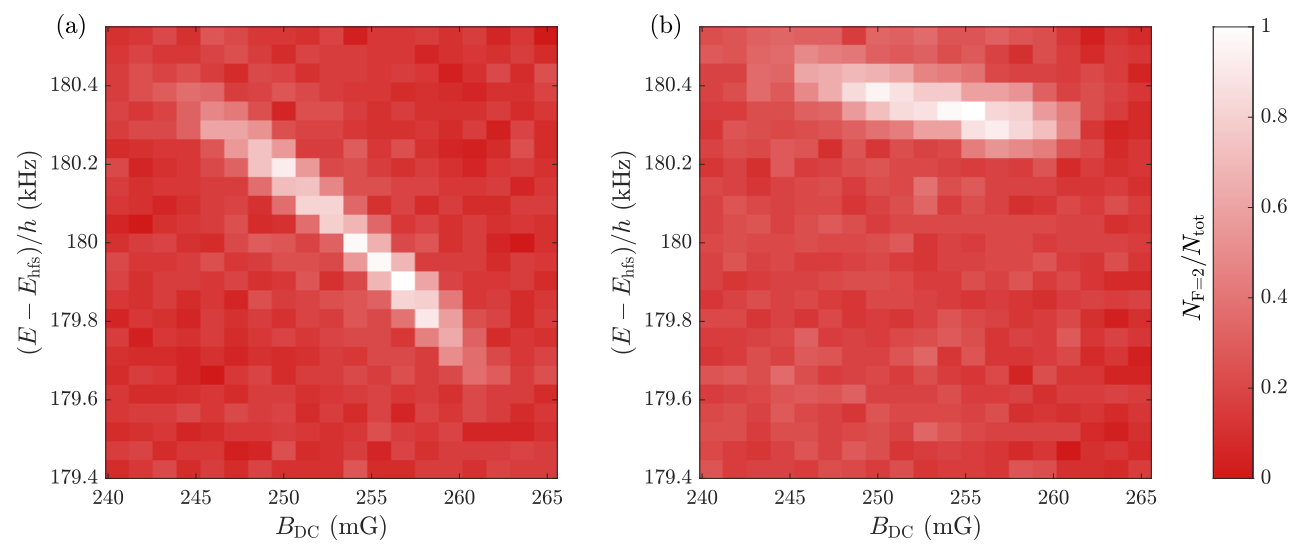

Figure 7. Cancelling field dependence with additional MW dressing. We prepare a pure RF-dressed $|1,-1\rangle$ state before applying a MW-pulse, scanning both the static field near RF resonance and the MW frequency around that of the transition $n=1,|1,-1\rangle \leftrightarrow|2,1\rangle$. The colour map denotes population transfer, scaling from dark red (all population in $F=1$ ) to white (all population in $F=2$ ). In (a) the resonance shows a significant dependence on the static field magnitude for no applied MW-dressing. In (b) data is shown for the same parameter range, but with additional MWdressing red-detuned from the $n=-2$ group. The flatness of the transition frequency is improved by a factor of 4 , limited by available MW power. The data in (a) corresponds to the frequency data in Fig. 6a. 
make the choice to alter the $|1,-1\rangle$ state by dressing on the $|1,-1\rangle \leftrightarrow|2,-2\rangle$ transition, as this is of the lowest frequency in any given group, and may therefore be reasonably far red-detuned without the concern of hitting other transitions in the potential landscape. We then choose a group to best fit the field dependence, ideally achieving $E_{(1,-1 \rightarrow 2,-2)}(B)=$ const $+E_{(1,-1 \rightarrow 2,1)}(B)-\partial \Omega_{\mathrm{MW}}^{2} / \partial B$, and observing any technical restrictions on microwave polarisation. The Rabi frequencies for the dressed transitions used here have been calculated ${ }^{21}$ and are plotted in Fig. 6b for the transition $|1,-1\rangle \leftrightarrow|2,-2\rangle$ in the groups $n=-3$ and $n=-2$ for $\sigma$ and $\pi$-polarised MW respectively. We see that these transitions have the opposite energy dependence on $B_{\mathrm{DC}}$ as our chosen pseudo-clock transition, and as such can have the effect of reducing the linewidth in a trapped scenario. For a full description of the effect, however, all dressed-state transitions and their appropriate detunings should be considered together with better approximations for the induced shifts.

Figure 7 shows experimental data for the application of this method. The spectrum near the targeted transition frequency is shown as a function of static field, with and without additional MW dressing. The data was taken by preparing a pure $|1,-1\rangle$ state and the color map indicates population transfer to $\mathrm{F}=2$. The presence of the additional MW field reduces the static field dependence over the region shown by a factor of 4, limited by available MW power. Technical improvement should reduce this further, and additional dressing frequencies could be applied to cancel higher order field dependencies, allowing for an almost arbitrarily flat transition.

\subsection{Removing trap asymmetry}

Figure 8a shows a two-dimensional representation of the potential landscape on the resonant torus, where the horizontal and vertical coordinates represent the toroidal (around the major radius) and poloidal (around the minor radius) angles respectively. Figure 8a shows non-isotropic traps that do not align with the poloidal and toroidal directions. The pattern exhibits a handedness that arises from the handedness of the RF-field configuration and changes sign with the $g_{\mathrm{F}}$-factor. In the harmonic approximation, the non-isotropy and misalignment can be analysed by expressing the curvature tensor at the trap minima. The squared trap frequencies are given by $\omega_{j k}^{2}=\left(\partial^{2} / \partial_{j} \partial_{k}\right) E_{m_{F}} / m_{0}$, where $m_{0}$ is the atomic mass of the trapped species. For simplicity, we assume real $a_{ \pm}$again and consider only the locally dominant field $u_{+}$or $u_{-}$being present. The curvature tensors for trap minima in top and bottom ring are given by

$$
\left.\omega_{j k}^{2}\right|_{\phi= \pm \frac{\pi}{2}}=\frac{\gamma}{m_{0}}\left(\begin{array}{ccc}
\frac{\delta^{2}+\frac{3}{2} a_{r}\left|u_{ \pm}\right|}{\rho_{0}^{2}} & \frac{l a_{z}\left|u_{ \pm}\right|}{r_{0} \rho_{0}} & 0 \\
\frac{l a_{z}\left|u_{ \pm}\right|}{r_{0} \rho_{0}} & \frac{l^{2} a_{r}\left|u_{ \pm}\right|}{r_{0}^{2}} & 0 \\
0 & 0 & 2 \sqrt{2} q^{2}
\end{array}\right)
$$

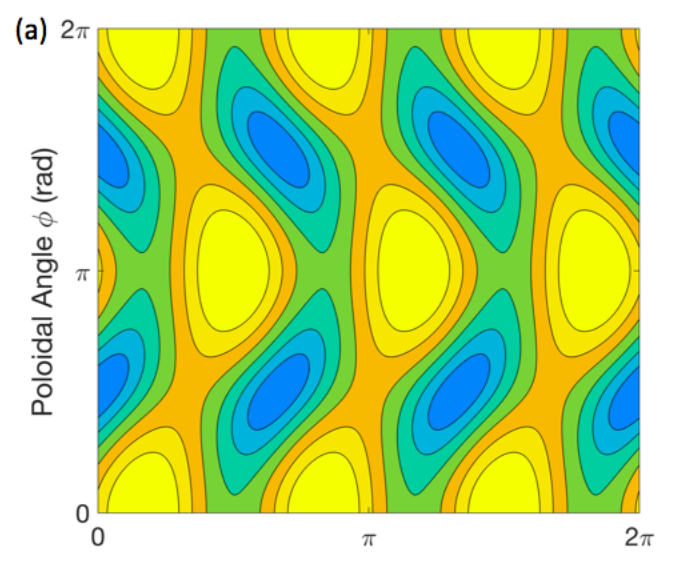

(b)

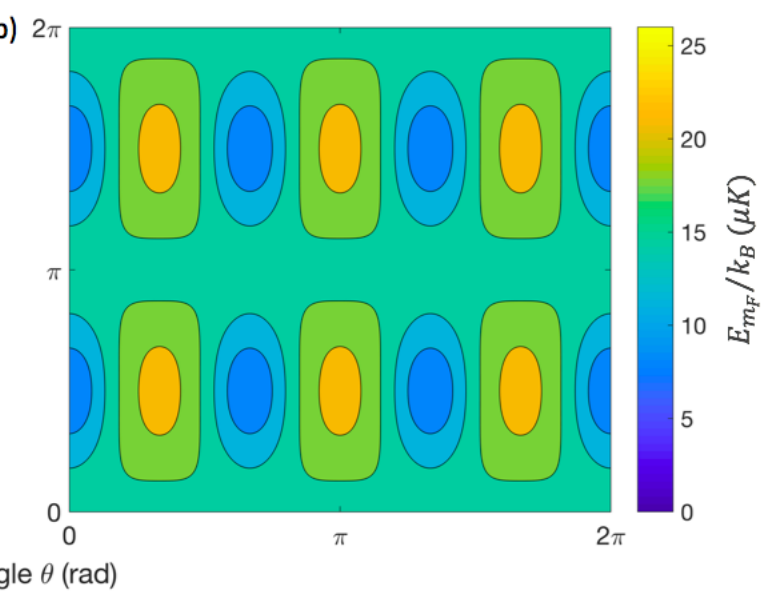

Figure 8. Removal of trap asymmetry via time averaging. In (a) the unmodulated RF-dressed potential is shown on the resonant surface, with corresponding parameters $a_{r}=0.9 \mathrm{G}, a_{z}=1.5 \mathrm{G},\left|u_{ \pm}\right|=0.3 \mathrm{G}$ and $l=3$. The time averaged adiabatic potential $V_{\text {avg }}$, with a modulation of $a_{z}(t)=a_{0} \cos \left(\omega_{m} t\right)$ is shown in (b). 
using the definitions $\delta^{2}=\left(a_{z}^{2}-a_{r}^{2}-\left|u_{ \pm}\right|^{2}\right) / \sqrt{2}, \gamma=m_{\mathrm{F}} g_{\mathrm{F}} \mu_{B} /\left(2 \sqrt{2} B_{0}\right)$, and the effective field strength at the extremum $B_{0}=\frac{1}{2}\left|a_{r}-\sqrt{2}\right| u_{ \pm}||$. The comparison of diagonal and off-diagonal elements shows that the sense of trap misalignment depends on the relative sign between amplitudes $a_{z}$ and $a_{r}$. The traps can be aligned, and the handedness can thus be removed, by modulating the sign of $a_{z}$ to form a time averaged dressed potential, ${ }^{15,26,27}$ which will make the time averaged curvature tensor diagonal in these coordinates. For practical purposes, it needs to be considered that the formation of time-averaged dressed potentials imposes some restrictions on the involved frequencies: $\omega_{0} \ll \omega_{m} \ll \Omega_{\mathrm{RF}} \ll \omega_{\mathrm{RF}}$. The modulation of the potential must be faster than the mechanical motion of the atoms at trap frequency $\omega_{0}$ but slower than the Rabi-frequency, which in turn must be lower than the dressing frequency for the RWA to be valid. This allows the atomic spin to follow the modulation whilst not exciting trap oscillations or transitions to other dressed states. In this scenario, these restriction become somewhat alleviated, because the Hamiltonian at the trap centres will remain unmodulated, where $a_{z}$ does not enter the coupling strength as given in Eq. 9 .

As an example, we assume a modulation $a_{z}(t)=a_{0} \cos \left(\omega_{m} t\right)$ to Eq. 3, where $\omega_{m}$ is the modulation frequency and $a_{0}=1.5 \mathrm{G}$ the magnitude of the modulation. The resulting potential is calculated as the average over a modulation period $T=2 \pi / \omega_{m}$

$$
V_{\mathrm{avg}}=\frac{1}{T} \int_{0}^{T} E_{\mathrm{F}, \bar{m}}(t) \mathrm{d} t .
$$

The result is shown in Fig. 8b. The trap misalignment has been removed, but comes with a certain shallowing of the potential landscape.

\section{EXPERIMENTAL REALISATION}

\subsection{Atom-chip setup}

We describe some of our technical development towards a fully trapped and guided Sagnac interferometer. For our purposes we require atom-chip technology for the generation of both the static magnetic field $\mathbf{B}_{\mathrm{DC}}$ and components of the RF-fields. The atom-chip assembly providing these fields is shown in Fig. 9. Two microfabricated substrates are stacked and bonded to a UHV-compatible, multi-layer printed circuit board (PCB), which comprises seven coils of various sizes to control the position of a magnetic trap during the initial loading and transport stage. The top-layer atom-chip generates a circular quadrupole field with a ring of zero field approximately $100 \mu \mathrm{m}$ from the surface. The atom-chip uses two layers of current carrying wires, because the necessary lead wires distort the ring quadrupole. Using the two-layer design, the generation of the static field can be switched between two coil sets, with lead wires at opposite feed points. Correctly timed, the switching between the coils will not affect the atom confinement or transport during the interferometer sequence as the atoms are transported around the ring track. The main RF-dressing field requires a strong radial component, which is generated with a micro-fabricated RF-coil. Two rectangular RF-coils have been included in the setup to provide the RF-fields to create a single trap, corresponding to multipole order $l=1$, i.e. the homogeneous interior dipole. Two coils are needed such that any arbitrarily polarised interior dipole can be created in the $x-y$ plane, which is necessary to compensate for small misalignment of the main RF-coil, and can be used to compensate for small tilts in the gravitational field. Due to physical constraints of the setup these RF-coils could not be placed perpendicular to each other. A monopole antenna next to the PCB provides the microwave field for the Ramsey sequence. The atom-chip assembly is mounted on a vacuum flange with electrical feedthroughs and heat sink, and placed upside down, compared to Figure 9, in a UHV chamber at a pressure $<10^{-10}$ mbar.

\subsection{Towards an RF-dressed ring trap}

We prepare an initial magneto-optical trap $(\mathrm{MOT})$ with $\approx 10^{8}$ atoms located approximately $2 \mathrm{~cm}$ underneath the PCB. The MOT setup uses three mutually orthogonal beam pairs, with a beam in the $z$-direction being reflected by a rear-side reflection-coated $\lambda / 4$ waveplate fixed on the surface of the PCB. The atomic cloud is compressed and cooled to a temperature of $\approx 70 \mu \mathrm{K}$ during an optical molasses stage, before optical pumping is applied to prepare the majority of atoms in the fully stretched $|2,2\rangle$ state. A magnetic quadrupole trap captures the ensemble, initially located $\approx 2 \mathrm{~cm}$ away from the center of the atom chip in the horizontal $x-y$ plane. The magnetic trap of lifetime $\approx 6.5 \mathrm{~s}$ is transported in $\approx 1 \mathrm{~s}$ towards the atom chip using the seven concentric $\mathrm{PCB}$ 

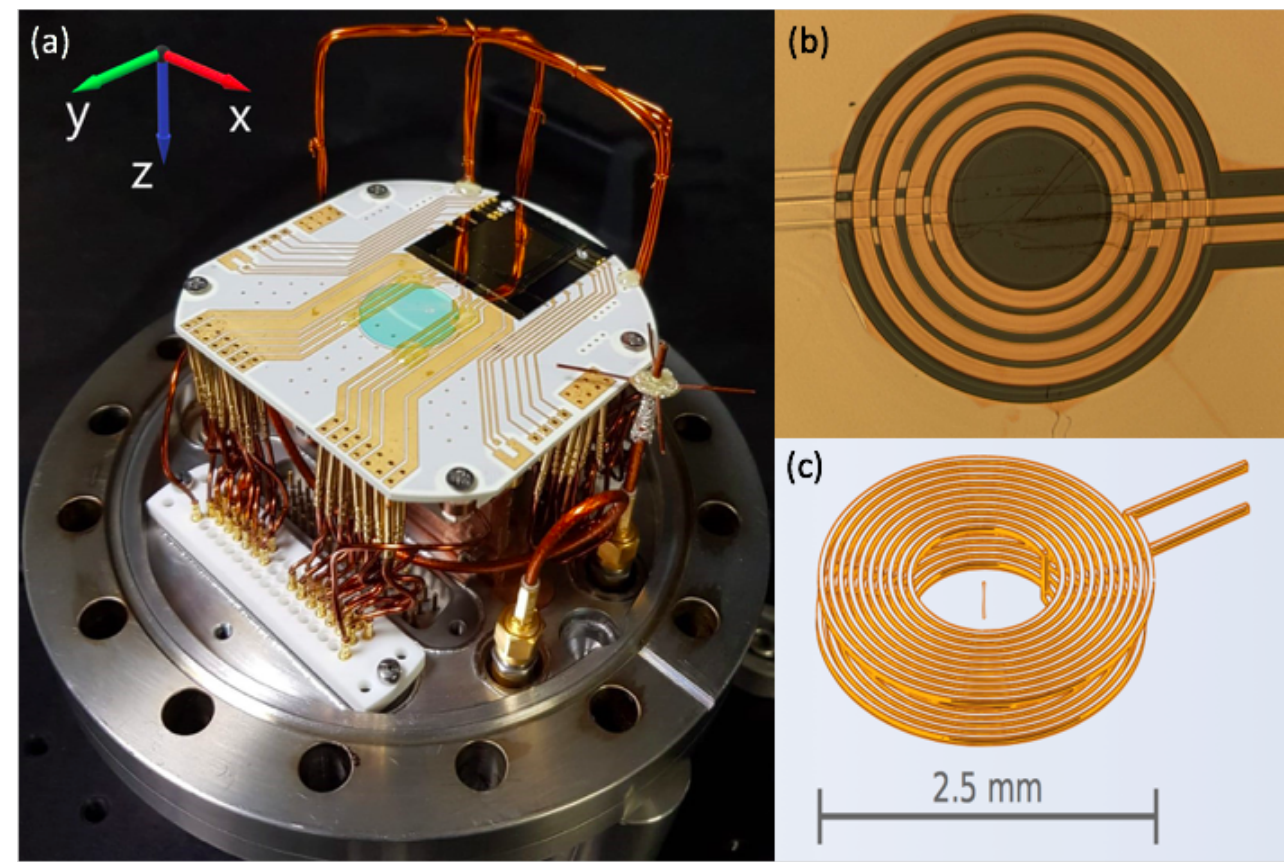

Figure 9. Our atom-chip assembly is shown in (a). A magneto-optical trap is created at about $2 \mathrm{~cm}$ distance from a surface mounted and rear coated $\lambda / 4$ waveplate (centre blue disk). Magnetically trapped atoms are then transported to two atom-chips (gold black squares) using a printed circuit board (white gold structure). Additional coils generate RFfields for atom transport around the ring. The microwave antenna (to the right of the PCB) creates a linearly polarised magnetic field in the $x-y$ plane. The entire assembly faces down when in the vacuum chamber. In (b) the central structure of the top-layer atom-chip is shown. Two closely spaced layers create a ring quadrupole of radius $r_{0}=0.5 \mathrm{~mm}$. The two layers produce identical fields away from the opposite feed points. A CAD drawing of the micro-fabricated RF-coil is shown in (c), which is placed between the top-layer atom-chip and the PCB.

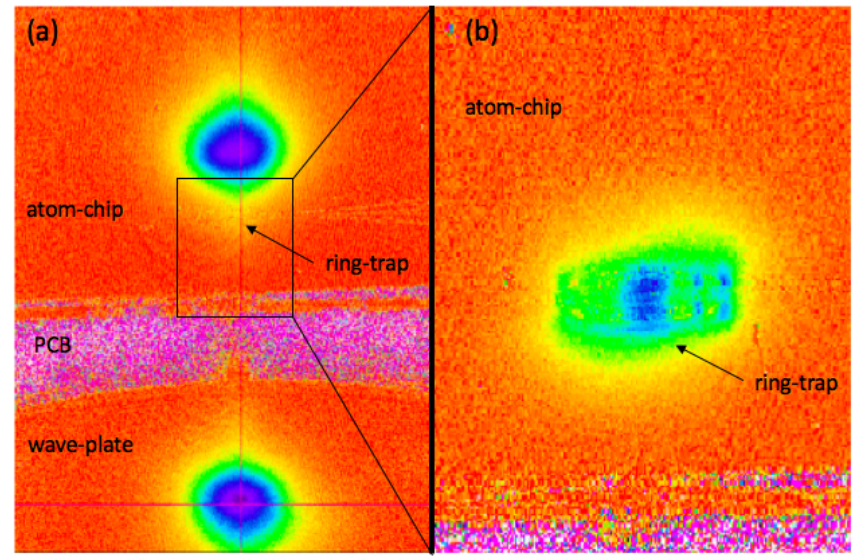

Figure 10. Loading of atoms into a ring-shaped trap. An imaging beam reflects off the atom-chip surface and the waveplate, resulting in a mirror image (top absorption image) and real image (bottom absorption image) of magnetically trapped atoms, as seen in (a). In (b) an expanded view of a loading attempt into a magnetic quadrupole ring trap is shown.

coils centred under the chip. The coils vary in size from $4.5 \mathrm{~cm}$ to $1 \mathrm{~mm}$ diameter to compress the cloud. The five largest coils require high currents of up to $10 \mathrm{~A}$ with 10 windings in respective PCB layers. The two smallest coils only use tracks on the surface of the $\mathrm{PCB}$ and support transport of the cloud to within $\approx 1 \mathrm{~mm}$ distance from the chip surface with $\approx 1.5 \mathrm{~A}$ currents. We transfer $\approx 10^{7}$ atoms to a ring-shaped quadrupole trap that is formed by four counterpropagating concentric chip currents, together with additional fields from PCB coils. The trap compression significantly heats the atomic cloud to $\approx 1 \mathrm{mK}$, and we initiate evaporative cooling during 
the final stages of the magnetic transport. The required RF-field is generated by the RF-chip coil that is placed between the top chip and the PCB and also used to create the RF-field described in Eq. 7. For atom clouds in the vicinity of the chip, analysis is performed by laser absorption imaging. The imaging beam is incident under a small glancing angle and reflected by the gold coated surface of the atom-chip as well as the waveplate on the PCB. The observed positions of real and mirror images and their point of convergence allow for controlled positioning of the trap directly below the atom-chip centre, see Fig. 10.

The next steps, further compression and adiabatic dressing, are currently under investigation. A radiofrequency ramp from low to high frequency will be used to form a dressed, toroidal trap, described in Sec. 3.2 above, where the target frequency $\omega_{R F}$ determines the size of the minor radius $\rho_{0}=\omega_{\mathrm{RF}} \hbar / q \mu_{\mathrm{B}} g_{\mathrm{F}}$.

\section{CONCLUSION}

In this paper we have outlined our progress on the development of a trapped Sagnac interferometer. A closed loop interferometer can be constructed with RF-dressed potentials that locally trap and state-dependently guide the atoms. This type of configuration could be used to remove wave-packet dispersion, gain control over cycle times under external acceleration, and increase the effective area of the interferometer. The necessary potentials are generated with atom-chip technology such that a robust portable device can be produced. Using hyperfine transitions between a trappable pair of RF-dressed pseudo-clock-states, we have demonstrated that a simple interferometric sequence is achievable. We have considered issues in coherence which emerge from mismatches in the trapping potentials of the two states and provided possible remedies to these problems. One of these issues is the discrepancy in the magnitude of the Landé $g_{\mathrm{F}}$ factors between the two states. Additional microwave dressing utilising the AC-Zeeman effect can be applied in order to cancel the mismatch in energy dependence between the two states. A second issue regarded the trap asymmetry, which may be corrected by an amplitude modulation of an RF dressing field component.

Currently, atoms have been loaded into the magnetic quadrupole ring trap, however certain issues still need to be overcome. The temperature of the atomic cloud needs to be further reduced via RF-evaporation, whilst still achieving an appropriate number of atoms after loading into the ring trap for dressing. After this, local trapping and state-dependent guiding of the atoms will follow, which should be a significant step towards realising Hafele and Keating's experiment on a chip.

\section{ACKNOWLEDGEMENTS}

We gratefully acknowledge the production of micro-fabricated atom chips by Ben Gurion University of the Negev, Be'er Sheva, Israel. Presented work was funded by the European Commission under project FP7-ICT-601180 (MatterWave), by EPSRC grant-no EP/M013294/1 (UK National Quantum Technology Hub in Sensors and Metrology) and by DARPA as part of the A-PhI programme (Atomic-Photonic Integration).

\section{REFERENCES}

[1] Clauser, J. F., "Ultra-high sensitivity accelerometers and gyroscopes using neutral atom matter-wave interferometry," Physica B+C 151(1-2), 262-272 (1988).

[2] Rosi, G., Cacciapuoti, L., Sorrentino, F., Menchetti, M., Prevedelli, M., and Tino, G. M., "Measurement of the gravity-field curvature by atom interferometry," Phys.Rev. Lett. 114(1), 013001 (2015).

[3] Riehle, F., Kisters, T., Witte, A., Helmcke, J., and Bordé, C. J., "Optical Ramsey spectroscopy in a rotating frame: Sagnac effect in a matter-wave interferometer," Phys. Rev. Lett. 67(2), 177 (1991).

[4] Gustavson, T. L., Landragin, A., and Kasevich, M. A., "Rotation sensing with a dual atom-interferometer Sagnac gyroscope," Classical and Quantum Gravity 17(12), 2385 (2000).

[5] Barrett, B., Geiger, R., Dutta, I., Meunier, M., Canuel, B., Gauguet, A., Bouyer, P., and Landragin, A., "The Sagnac effect: 20 years of development in matter-wave interferometry," C. R. Phys. 15(10), 875-883 (2014).

[6] Bell, T., Glidden, J. A. P., Humbert, L., Bromley, M. W. J., Haine, S. A., Davis, M. J., Neely, T. W., Baker, M. A., and Rubinsztein-Dunlop, H., "Bose-Einstein condensation in large time-averaged optical ring potentials," New J. Phys. 18(3), 035003 (2016). 
[7] Stevenson, R., Hush, M. R., Bishop, T., Lesanovsky, I., and Fernholz, T., "Sagnac interferometry with a single atomic clock," Phys. Rev. Lett. 115(16), 163001 (2015).

[8] Pandey, S., Mas, H., Drougakis, G., Thekkeppatt, P., Bolpasi, V., Vasilakis., G., Poulios, K., and von Klitzing, W., "Hypersonic Bose-Einstein condensates in accelerator rings," Nature 570, 205-209 (2019).

[9] Post, E. J., "Sagnac effect," Rev. of Mod. Phys. 39(2), 475 (1967).

[10] Malykin, G. B., "The Sagnac effect: correct and incorrect explanations," Physics-Uspekhi 43(12), 1229 (2000).

[11] Dieks, D. and Nienhuis, G., "Relativistic aspects of nonrelativistic quantum mechanics," Am. J. Phys. 58(7), 650-655 (1990).

[12] Lan, S.-Y., Kuan, P.-C., Estey, B., English, D., Brown, J. M., Hohensee, M. A., and Müller, H., "A clock directly linking time to a particle's mass," Science 339, 554-557 (2013).

[13] Hafele, J. and Keating, R., "Around the world atomic clocks: observed relativistic time gains," Science 177, 168 (1972).

[14] Zhou, Y., Lesanovsky, I., Fernholz, T., and Li, W., "Controlling the dynamical scale factor in a trapped atom Sagnac interferometer," (2018). preprint, arXiv:1811.11107.

[15] Navez, P., Pandey, S., Mas, H., Poulios, K., Fernholz, T., and von Klitzing, W., "Matter-wave interferometers using TAAP rings," New J. Phys. 18(7), 075014 (2016).

[16] Szmuk, R., Dugrain, V., Maineult, W., Reicheland, J., and Rosenbusch, P., "Stability of a trapped-atom clock on a chip," Phys. Rev. A 92, 012106 (2015).

[17] Ammar, M., Dupont-Nivet, M., Huet, L., Pocholle, J.-P., Rosenbusch, P., Bouchoule, I., Westbrook, C. I., Estève, J., Reichel, J., Guerlin, C., and Schwartz, S., "Symmetric microwave potentials for interferometry with thermal atoms on a chip," Phys. Rev. A 91, 053623 (2015).

[18] Fernholz, T., Krauter, H., Jensen, K., Sherson, J. F., Sørensen, A. S., and Polzik, E. S., "Spin squeezing of atomic ensembles via nuclear-electronic spin entanglement," Phys. Rev. Lett. 101, 073601 (2008).

[19] Gentile, F., Johnson, J., Poulios, K., and Fernholz, T., "Ring-shaped atom-trap lattices using multipole dressing fields," (2019). preprint, arXiv:1909.01186.

[20] Jammi, S., Pyragius, T., Bason, M. G., Florez, H. M., and Fernholz, T., "Dispersive detection of radiofrequency-dressed states," Phys. Rev. A 97, 043416 (2018).

[21] Sinuco-Leon, G. A., Garraway, B. M., Mas, H., Pandey, S., Vasilakis, G., Bolpasi, V., von Klitzing, W., Foxon, B., Jammi, S., Poulios, K., and Fernholz, T., "Microwave spectroscopy of radio-frequency-dressed ${ }^{87} \mathrm{Rb}, "$ Phys. Rev. A 100, 053416 (2019).

[22] Garraway, B. M. and Perrin, H., "Recent developments in trapping and manipulation of atoms with adiabatic potentials," J. Phys. B: At. Mol. Opt. 49(17), 172001 (2016).

[23] Gentile, F., Towards an atomic Sagnac interferometer with full dynamical control of atoms in ring waveguides, PhD thesis, The University of Nottingham, Nottingham (2019).

[24] Kazakov, G. and Schumm, T., "Magic radio-frequency dressing for trapped atomic microwave clocks," Phys. Rev. A 91, 023404 (2015).

[25] Sarkany, L., Weiss, P., Hattermann, H., and Fortágh, J., "Controlling the magnetic field sensitivity of atomic clock states by microwave dressing," Phys. Rev. A 90, 053416 (2014).

[26] Lesanovsky, I. and von Klitzing, W., "Time-averaged adiabatic potentials: Versatile matter-wave guides and atom traps," Phys. Rev. Lett. 99(8), 083001 (2007).

[27] Sherlock, B. E., Gildemeister, M., Owen, E., Nugent, E., and Foot, C. J., "Time-averaged adiabatic ring potential for ultracold atoms," Phys. Rev. A 83(4), 043408 (2011). 